\title{
On the integration of low frequency transformer into modular multilevel converter
}

A. Christe and D. Dujić

This material is posted here with permission of the IEEE. Such permission of the IEEE does not in any way imply IEEE endorsement of any of EPFL's products or services. Internal or personal use of this material is permitted. However, permission to reprint / republish this material for advertising or promotional purposes or for creating new collective works for resale or redistribution must be obtained from the IEEE by writing to pubs-permissions@ieee. org. By choosing to view this document, you agree to all provisions of the copyright laws protecting it. 


\title{
On the Integration of Low Frequency Transformer into Modular Multilevel Converter
}

\author{
Alexandre Christe and Dražen Dujić \\ Power Electronics Laboratory - PEL \\ École Polytechnique Fédérale de Lausanne - EPFL \\ Station 11, CH-1015 Lausanne \\ alexandre.christe@epfl.ch,drazen.dujic@epfl.ch
}

\begin{abstract}
Various high power applications require power converters with large voltage step ratios, easily achieved by inclusion of a single or multiple transformers, which provide galvanic isolation at the same time. This paper presents, in a systematic manner, the necessary steps for the integration of a Low Frequency Transformer (LFT) into the Modular Multilevel Converter (MMC). Unlike the classical MMC that requires an external transformer for galvanic isolation, this work considers a transformer integration at the arm level resulting in a complete replacement of the arm inductors. Such galvanically isolated modular converters can be realized either in interleaved or stacked arrangements. The properties of each variant are discussed and compared with the classical MMC with external LFT, on the system design level but also from the control point of view.
\end{abstract}

\section{INTRODUCTION}

The Modular Multilevel Converter (MMC) [1] is one of the most active research topic in power electronics for the last decade. This is especially true for the HVDC applications where it has been adopted and commercialized rather quickly by the industry. The main advantages of MMC are the absence of series-connected devices compared to classical Voltage Source Converters (VSCs), almost unlimited voltage scalability by means of series-connected submodules (SM), opportunity to standardize SM and use in variety of applications, the reduction or cancellation of filtering needs due to increased quality of AC voltage waveform, etc. This is possible at the expense of increased control effort, communication complexity and overall converter volume increase, compared to the equivalent state-of-the-art counterparts.

In MVDC applications, MMC has been considered for drive applications, even though low-frequency operation is not most favorable [2], [3], or so-called shaft-generator applications [4] where the MMC is used as an inverter to supply MVAC onboard distribution system. Smart grid applications have seen an increased interest into Solid State Transformers (SSTs) [5], where multiple medium frequency transformers are used for galvanic isolation, while multi-stage power conversion offers increased control and power quality features.

The work presented in this paper considers applications where two grids with different voltage levels have to be interfaced (e.g. MVDC grid and LVAC grid). Rather than relying on some variant of the SST, a single-stage conversion realized through combination of the MMC and an LFT is considered and analyzed. The use of a transformer allows to deal with large voltage step ratio between the two grids. From an efficiency point of view, it would be advantageous compared to the efficiency figures reported for various SSTs. To achieve better integration at the system level, the LFT is integrated into the arms of the MMC. Several publications have been addressing this topic already [6]-[11], but so far no comparative or systematic assessment has been performed. For the first time, this is presented and demonstrated by means of extensive theoretical development and simulation results.

This paper is organized as follows: in Section 2 a reference case is established based on the classical MMC in combination with external LFT. Section 3 provides a review of the previous efforts in this field and systematically derives two basic MMC arm variants with integrated transformer winding. The modeling of the different topological variants is presented in Section 4, in order to highlight similarities and differences from the control point of view. A simplified control system design is presented in Section 5, and comparative performance assessment by means of numerical simulations is presented in Section 6. Summary and concluding remarks are provided in Section 7.

\section{MMC WITH EXTERNAL LFT}

Classical MMC with external LFT is considered as a reference case for the benchmark. Each arm comprises of $N_{s m}$ series-connected unipolar SMs with separate (discrete) arm inductors. The MVDC voltage on the DC side is equally split between two voltage sources, allowing a connection to either be a unipolar or a bipolar transmission. The LFT is connected to the phase leg midpoint terminals in a star configuration. For simplicity reasons, unitary turns ratio of the LFT is considered for the analysis, even though step-down of voltage by means of LFT would be used in real application. The representation used for modeling with all relevant variables is shown in Fig. 1.

The development of a decoupled model (power decoupling between the $\mathrm{DC}$ and the $\mathrm{AC}$ terminals), similarly to that reported in [12], and the control structure will be presented in section 4 . The choice of the harmonic content of the circulating current (also called partial bus current or differential current) is 
free to select, as long as the harmonic spectrum of the grid current comprises only of a fundamental component. In particular, a second harmonic component may be intentionally injected in the arm currents in order to reduce the capacitive storage requirements [13], [14] or [15]. The modulation functions for each arm are complementary since each arm has to block the full input DC link voltage, and are given as:

$$
\begin{aligned}
& m_{p}(t)=\frac{1}{2}(1-m \sin (\omega t-\pi)) \\
& m_{n}(t)=\frac{1}{2}(1+m \sin (\omega t-\pi))
\end{aligned}
$$

The combination of the MMC and an external LFT still requires passive components (i.e. arm inductors) to be added into the converter structure, which may occupy a significant part of the total volume of the converter. The work presented in this paper aims to explore possibilities to replace the arm inductors by means of integration of the transformer winding directly into the arms of the MMC.

\section{INTEGRATION OF THE LFT INTO THE MMC ARM}

Integration of the winding of a transformer into the MMC arm has already been addressed in a few publications [6]-[8], [10], [11]. These are briefly reviewed here.

\section{A. Preliminary considerations}

The most straightforward integration of the LFT at the arm level was proposed (also patented) in [8], [9]. The principles are borrowed from the open-end winding machine drives, and the primary winding of the LFT is directly connected between two sets of SMs creating the MMC arms. Its schematic is presented in Fig. 2. This topology will be referred as the OpenEnd Winding MMC (OEWMMC).

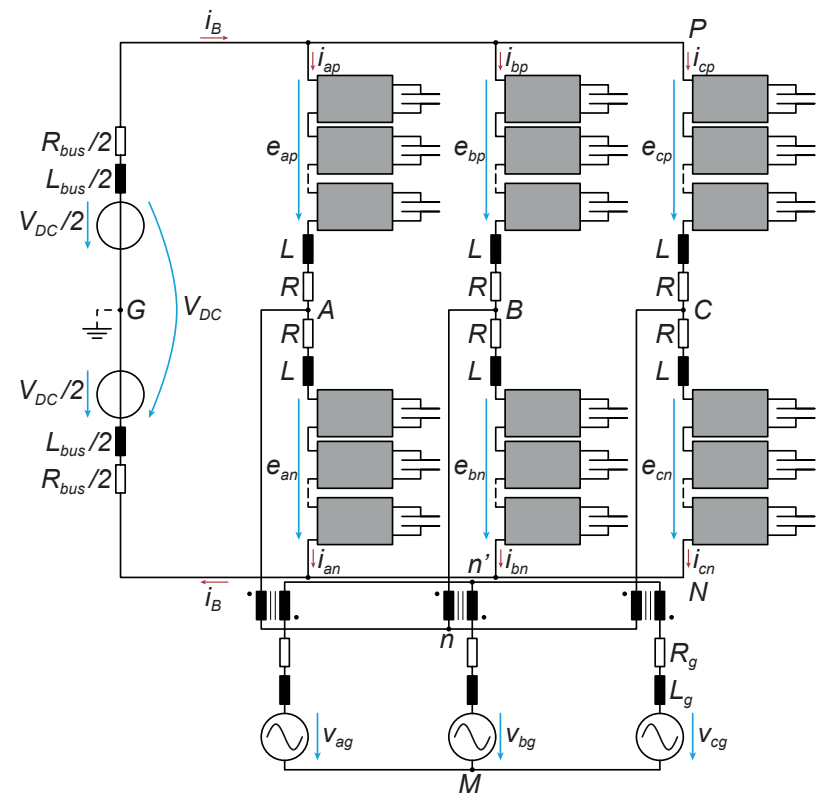

Fig. 1. Classical MMC with external LFT.
Such an integration implies changes at the modulation level, as the grid voltage will be reflected to the voltage across the primary side of the transformer. In the classical MMC case, each arm has to block the full DC link voltage, but in the OEWMMC case, both arms are modulated with the same modulation function:

$$
m_{p}(t)=m_{n}(t)=\frac{1}{2}(1-m \sin (\omega t-\pi))
$$

The voltage swing across the integrated transformer's primary winding is with amplitude equal, at most, to the full DC link voltage (H-bridge like operation), which is doubled compared to the classical MMC case (the phase leg midpoint swings between $P$ and $N$, so that the maximum amplitude is half the DC link voltage). This extended operation is achieved without increasing the arms blocking voltage. It has been shown in [16] that the OEWMMC suffers from DC bias in the transformer, resulting in poor magnetic material utilization and questioning the feasibility for real implementation, as the LFT would have to be significantly oversized. Due to the transformer arrangement in the OEWMMC, one third of the DC current has to flow through the integrated winding, contributing to the DC flux component of the transformer.

Consequently, the OEWMMC doesn't match the requirement of power decoupling like the classical MMC, because the two arms are not really independent one from another $\left(i_{x p}\right.$ and $i_{x n}$ are strictly identical, $\left.x \in\{a, b, c\}\right)$.

Another attempt at integrating the transformer at the arm level was done (also patented) in [7]. The main proposition was however targeting two ports isolated DC/DC conversion and relies also on use of a single arm per phase leg. The problem of the magnetic DC flux offset has been recognized and considered in the proposal of the elementary structure. In order to prevent AC currents to flow through the DC terminals of the converter, the minimal configuration comprises two

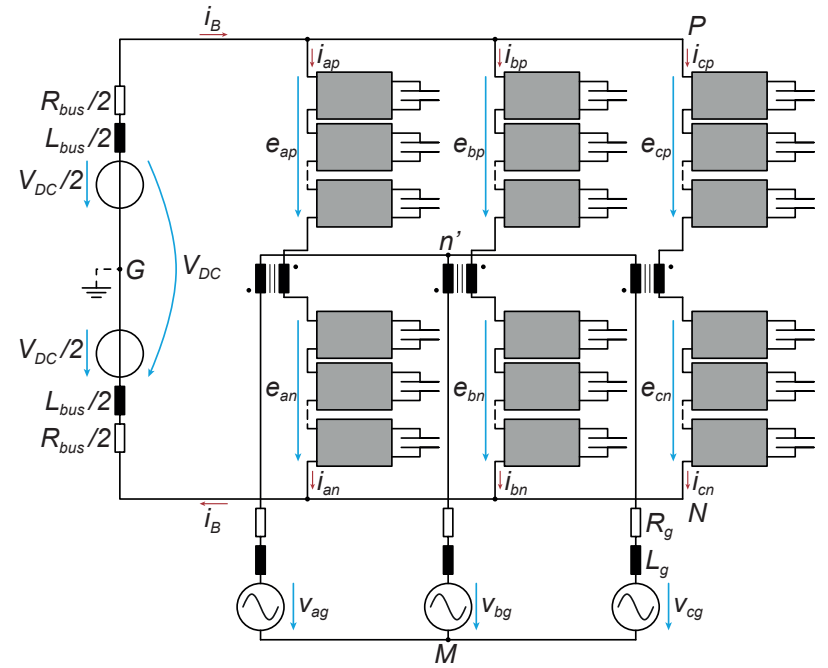

Fig. 2. Open-end windings MMC proposed in [8], [9]. 
phase legs per each DC side. For that reason, each pair of arms are modulated in opposite phase:

$$
m_{l}(t)=m \sin (2 \pi f t-\pi) \quad m_{r}(t)=m \sin (2 \pi f t)
$$

where $f$ is the frequency of the $\mathrm{AC}$ component. The blocking capability of each arm is directly linked to the peak-topeak value of the modulation function. The series-connected unipolar submodules have to block $2 V_{D C}$. The magnetic coupling is done according to the double lines in Fig. 3, where each winding of $D C_{1}$ is coupled with one winding of $D C_{2}$. As $i_{B, 1}$ and $i_{B, 2}$ are always of opposite polarity, the DC flux cancellation inside the magnetic material is achieved independently of the operating point.

Even if the representation of one phase leg of the MMC proposed in [7] or [11] seems to be apparently different from one phase leg of the OEWMMC proposed in Fig. 2, this comes from the fact that they rely on two different perceptions of the same thing. In other words, there are not two separate arms per phase leg. Consequently, no vertical balancing will be present for the OEWMMC, as in order not to be seen at the AC terminals, it is done by vertical shifting of the transformer windings, i.e. by playing on the DC part of the modulation function (which corresponds to the arm balancing method for PS-PWM) or correctly choosing which SMs have to be inserted or bypassed at a given time without affecting the sum of inserted / bypassed SMs (which corresponds to the arm balancing method based on a sorting algorithm).

\section{B. MMC phase leg structures with integrated LFT}

Regarding the previous propositions, fundamental properties that should be fulfilled for a DC-free transformer operation, can be highlighted. The inherent DC current flowing through the MMC phase legs is responsible for the active power transfer. Thus, it is not possible to act on it by control means. So any DC bias prevention in the magnetic material has to be performed inside the magnetic structure itself by a suitable windings polarity arrangement. In order to prevent the presence of AC content at the DC terminals, there should be

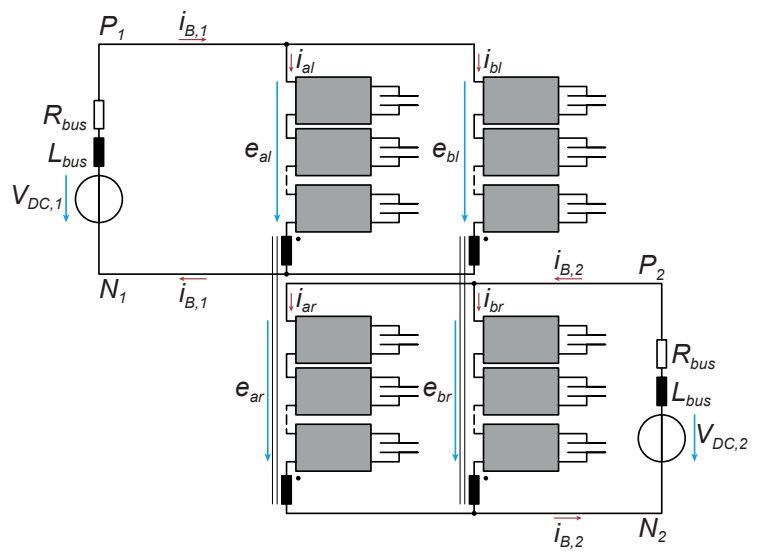

Fig. 3. Two port isolated MMC proposed in [7].

at least two arms per phase leg. In the simplest case, a singlephase DC/AC conversion will be performed. The real elementary stage, able to perform direct DC/AC conversion with a single DC input, could be as well extended to any number of phases by paralleling elementary stages. The elementary stage is presented in Fig. 4 (a). It is worth mentioning that from a transformer design point of view, the winding series connection on the primary side has to withstand a full DC link voltage. A similar elementary stage is used in [6] under the name Push-Pull MMC. The authors targeted battery energy storage system application, that features a large voltage step ratio between the two sources.

In addition to the interleaved case, the elementary stage could also be used in a stacked fashion. A similar configuration was reported in the work of [10] under the name ThreeWindings MMC. Its schematic is presented in Fig. 4 (b). The equivalent scheme for the magnetic structure is represented on Fig. 5.

\section{MMC MODELING}

\section{A. Transformer model}

In all further developments, a simplified "L" transformer model will be used (Fig. 6), where only one leakage path is present. It is particularly beneficial from a control point of view, as it allows to reduce the number of state variables. As the focus of this paper is not on the magnetic design of the LFT, this is an acceptable simplification. The usual conventions for transformer are followed in this paper. In the next developments, all equations are referred to the primary

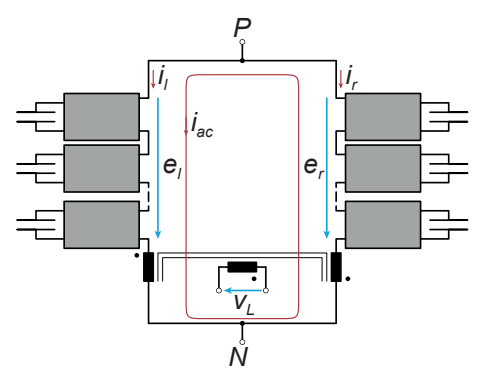

(a)

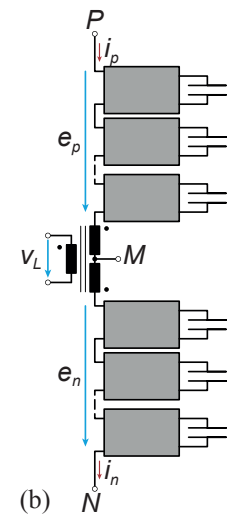

Fig. 4. Elementary stages with DC cancellation in the magnetic structure: (a) interleaved and (b) stacked where $M$ creates a neutral with the other phases.

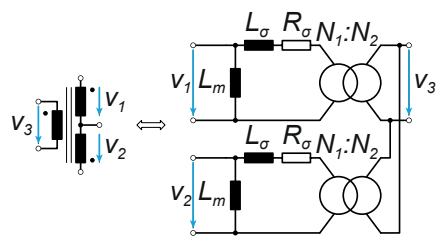

Fig. 5. Equivalent scheme of the dual primaries transformer with DC cancellation. 
side. The turns ratio is defined as $n=N_{2} / N_{1}$, which means $N_{1}: N_{2}$ corresponds to $1: n$, where $n$ is not limited to the positive integers. The reported quantities to the primary side are defined as:

$$
V^{\prime}=\frac{V}{n} \quad I^{\prime}=n I \quad Z^{\prime}=\frac{Z}{n^{2}}
$$

According to Fig. 6, the leakage components have the following values:

$$
L_{\sigma}=L_{\sigma 1}+\frac{L_{\sigma 2}}{n^{2}} \quad R_{\sigma}=R_{\sigma 1}+\frac{R_{\sigma 2}}{n^{2}}
$$

whose are valid under the assumption $L_{m} \gg L_{\sigma}$.

\section{B. External quantities}

For all three topologies, the dynamics of the external quantities (DC bus side and grid side) are common. KVL equations will account afterwards for them through their terminal voltages, namely $v_{B}$ and $v_{L}$. One has:

$$
\begin{aligned}
v_{\text {bus }} & =R_{\text {bus }} i_{B}+L_{\text {bus }} \frac{\mathrm{d}}{\mathrm{d} t} i_{B}+v_{B} \\
v_{\text {bus }} / 2 & =R_{\text {bus }} / 2 i_{B}+L_{b u s} / 2 \frac{\mathrm{d}}{\mathrm{d} t} i_{B}+v_{B} \\
v_{L} & =R_{g} i_{L}+L_{g} \frac{\mathrm{d}}{\mathrm{d} t} i_{L}+v_{g}
\end{aligned}
$$

In particular, the line quantities will be reported to the primary side of the transformer:

$$
v_{L}^{\prime}=\frac{R_{g}}{n} i_{L}+\frac{L_{g}}{n} \frac{\mathrm{d}}{\mathrm{d} t} i_{L}+\frac{v_{g}}{n}
$$

The external circuits are presented in Fig. 7.

\section{Classical MMC}

The modeling of the classical MMC has been extensively reported in the literature [12], [17], [18]. Most of them are based on a simplified single-phase model (cf. Fig. 8) that is then extended to the specific number of phases according to the application. The most comprehensive way to account at the

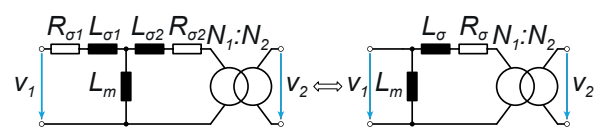

Fig. 6. Transformer model adopted in the paper.

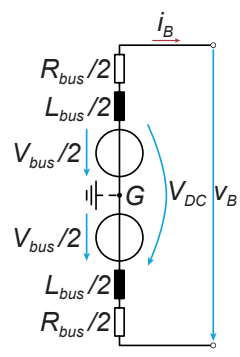

(a)

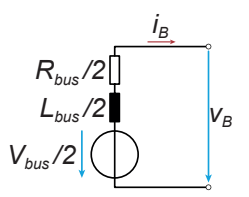

(b)

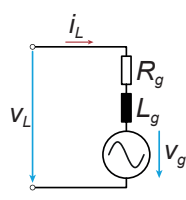

(c)
Fig. 7. External circuits: (a) DC link for classical MMC and stacked stage, (b) DC link for interleaved stage and (c) AC grid. same time for the arm current and summed capacitor voltage dynamic relies on a pair of controlled current / voltage sources. Two equivalent variables have to be defined: $C_{a r m}$ for the series-connection of $N_{s m}$ times the submodule capacitor and similarly $R_{e q}$. Please note that $R_{e q}$ is mandatory in order to get invertible matrices.

$$
C_{a r m}=\frac{C_{s m}}{N_{s m}} \quad R_{e q}=N_{s m} R_{s m}
$$

The average arm dynamics are the following:

$$
e_{p}=m_{p} V_{p}^{\Sigma} \quad e_{n}=m_{n} V_{n}^{\Sigma} \quad i_{p}^{\Sigma}=m_{p} i_{p} \quad i_{n}^{\Sigma}=m_{n} i_{n}
$$

The external circuit for the DC source (Fig. 7 (a)) will be connected between $P$ and $N$. The voltage across its terminals is defined as $v_{B}$. According to $\mathrm{KVL}$, the following "DC" loop is defined:

$$
v_{B}=e_{p}+e_{n}+R\left(i_{p}+i_{n}\right)+L\left(\frac{\mathrm{d}}{\mathrm{d} t} i_{p}+\frac{\mathrm{d}}{\mathrm{d} t} i_{n}\right)
$$

Equivalently, $v_{L}$ is defined as the terminal voltage where the AC grid external circuit (Fig. 7 (c)) will be connected.

$$
\begin{aligned}
v_{L}^{\prime}= & \frac{-e_{p}+e_{n}}{2}-\frac{R}{2}\left(i_{p}-i_{n}\right)-\frac{L}{2}\left(\frac{\mathrm{d}}{\mathrm{d} t} i_{p}-\frac{\mathrm{d}}{\mathrm{d} t} i_{n}\right) \\
& -R_{\sigma} i_{L}-L_{\sigma} \frac{\mathrm{d}}{\mathrm{d} t} i_{L} \\
v_{L}^{\prime}= & -R_{\sigma} i_{L}-L_{\sigma} \frac{\mathrm{d}}{\mathrm{d} t} i_{L}+L_{m} \frac{\mathrm{d}}{\mathrm{d} t} i_{m}
\end{aligned}
$$

The two equations, above, represent two parallel branches, the main branch and the magnetizing branch, and thus shouldn't be combined into one equation. Alternate variables might be introduced:

$$
\begin{array}{ll}
v_{B}=v_{p}+v_{n} & v_{L}^{\prime}=\frac{-v_{p}+v_{n}}{2} \\
e_{B}=e_{p}+e_{n} & e_{L}=\frac{-e_{p}+e_{n}}{2} \\
i_{B}=\frac{i_{p}+i_{n}}{2} & i_{L}=i_{p}-i_{n}-i_{m}
\end{array}
$$

The decoupling in the obtained model is illustrated by no coupling between $i_{B}$ and $i_{L}, i_{m}$. Those transformations may result into Fig. 9, where the decoupling between the DC and

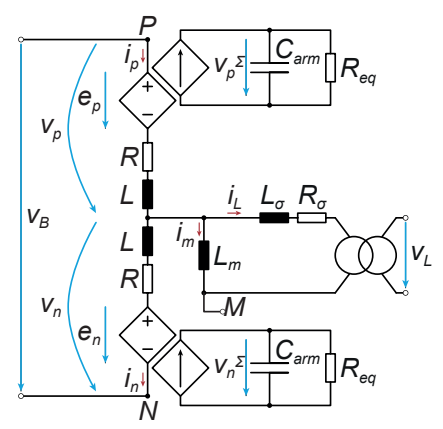

Fig. 8. Classical MMC phase with equivalent transformer circuit and variables for modeling. $M$ is the transformer's primary side star connection. 
AC quantities is now obvious. This offers to control them independently.

$$
\begin{aligned}
{\left[\begin{array}{c}
v_{B} \\
v_{L}^{\prime} \\
v_{L}^{\prime}
\end{array}\right]=} & {\left[\begin{array}{c}
e_{B} \\
e_{L} \\
0
\end{array}\right]+\left[\begin{array}{ccc}
2 R & 0 & 0 \\
0 & -R / 2-R_{\sigma} & -R / 2 \\
0 & -R_{\sigma} & 0
\end{array}\right]\left[\begin{array}{c}
i_{B} \\
i_{L} \\
i_{m}
\end{array}\right] } \\
& +\left[\begin{array}{ccc}
2 L & 0 & 0 \\
0 & -L / 2-L_{\sigma} & -L / 2 \\
0 & -L_{\sigma} & L_{m}
\end{array}\right] \frac{\mathrm{d}}{\mathrm{d} t}\left[\begin{array}{c}
i_{B} \\
i_{L} \\
i_{m}
\end{array}\right]
\end{aligned}
$$

\section{Interleaved stage}

According to Fig. 10, the average model for the interleaved stage can be established. The average dynamics of the arm are the following:

$$
e_{l}=m_{l} V_{l}^{\Sigma} \quad e_{r}=m_{r} V_{r}^{\Sigma} \quad i_{l}^{\Sigma}=m_{l} i_{l} \quad i_{r}^{\Sigma}=m_{r} i_{r}
$$

When encompassing the DC link quantities, one has:

$$
v_{B}=e_{l}+L_{m} \frac{\mathrm{d}}{\mathrm{d} t} i_{m l}=e_{r}+L_{m} \frac{\mathrm{d}}{\mathrm{d} t} i_{m r}
$$

It is further simplified to:

$$
v_{B}=\frac{e_{l}+e_{r}}{2}+\frac{L_{m}}{2}\left(\frac{\mathrm{d}}{\mathrm{d} t} i_{m l}+\frac{\mathrm{d}}{\mathrm{d} t} i_{m r}\right)
$$

Similarly, with respect to the line voltage:

$$
\begin{aligned}
2 v_{L}= & -R_{\sigma} i_{o l}-L_{\sigma} \frac{\mathrm{d}}{\mathrm{d} t} i_{o l}-e_{l}+e_{r}+R_{\sigma} i_{o r} \\
& +L_{\sigma} \frac{\mathrm{d}}{\mathrm{d} t} i_{\text {or }} \\
2 v_{L}= & -R_{\sigma} i_{o l}-L_{\sigma} \frac{\mathrm{d}}{\mathrm{d} t} i_{o l}+L_{m} \frac{\mathrm{d}}{\mathrm{d} t} i_{m l}-L_{m} \frac{\mathrm{d}}{\mathrm{d} t} i_{m r} \\
& +R_{\sigma} i_{o r}+L_{\sigma} \frac{\mathrm{d}}{\mathrm{d} t} i_{o r}
\end{aligned}
$$

Fig. 9. Equivalent decoupled model for the classical MMC including LFT.

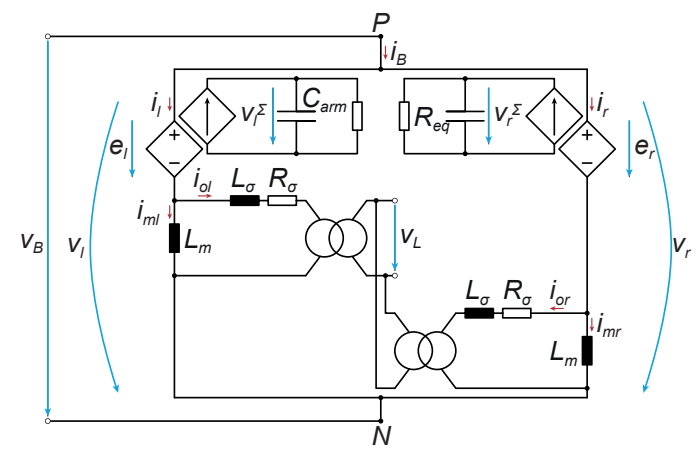

Fig. 10. Interleaved MMC stage with equivalent transformer circuit and variables for modeling.
Several new variables might be advantageously introduced for simplifying the notation:

$$
\begin{aligned}
& v_{B}=\frac{v_{l}+v_{r}}{2} \quad v_{L}=\frac{-v_{l}+v_{r}}{2} \\
& e_{B}=\frac{e_{l}+e_{r}}{2} \quad e_{L}=\frac{-e_{l}+e_{r}}{2} \\
& i_{B}=i_{l}+i_{r} \quad i_{L}=i_{l}-i_{r}-i_{m}=i_{\text {ol }}-i_{\text {or }}
\end{aligned}
$$

From Eq. (18c), the magnetizing currents cancel each other for the bus current $\left(i_{m l}+i_{m r}\right)$, while they sum up for the line current $\left(i_{m l}-i_{m r}\right)$. This means no $\mathrm{AC}$ is transferred to the DC link, and that the DC fluxes compensate in the magnetic structure. This modeling step allows the deduction of a decoupled equivalent circuit in Fig. 11, with the transformed variables as defined in Eq. (18).

$$
\begin{aligned}
{\left[\begin{array}{c}
v_{B} \\
v_{L}^{\prime} \\
v_{L}^{\prime}
\end{array}\right]=} & {\left[\begin{array}{c}
e_{B} \\
e_{L} \\
0
\end{array}\right]+\left[\begin{array}{ccc}
L_{m} / 2 & 0 & 0 \\
0 & -R_{\sigma} / 2 & 0 \\
0 & -R_{\sigma} / 2 & 0
\end{array}\right]\left[\begin{array}{c}
i_{B} \\
i_{L} \\
i_{m}
\end{array}\right] } \\
& +\left[\begin{array}{ccc}
L_{m} / 2 & 0 & 0 \\
0 & -L_{\sigma} / 2 & 0 \\
0 & -L_{\sigma} / 2 & L_{m} / 2
\end{array}\right] \frac{\mathrm{d}}{\mathrm{d} t}\left[\begin{array}{c}
i_{B} \\
i_{L} \\
i_{m}
\end{array}\right]
\end{aligned}
$$

\section{E. Stacked stage}

According to Fig. 12, the average model for the stacked stage can be established. As for the interleaved stage, the arms have been replaced by their average equivalents and $C_{a r m}$ plus $R_{e q}$ share the same definitions.

$$
e_{p}=m_{p} V_{p}^{\Sigma} \quad e_{n}=m_{n} V_{n}^{\Sigma} \quad i_{p}^{\Sigma}=m_{p} i_{p} \quad i_{n}^{\Sigma}=m_{n} i_{n}
$$

When encompassing the DC link quantities, one has:

$$
\begin{aligned}
v_{B} & =e_{p}+L_{m} \frac{\mathrm{d}}{\mathrm{d} t} i_{m p}+e_{n}+L_{m} \frac{\mathrm{d}}{\mathrm{d} t} i_{m n} \\
& =e_{p}+e_{n}+L_{m}\left(\frac{\mathrm{d}}{\mathrm{d} t} i_{m p}+\frac{\mathrm{d}}{\mathrm{d} t} i_{m n}\right)
\end{aligned}
$$

Similarly, w.r.t. the line voltage:

$$
\begin{aligned}
v_{L} & =-R_{\sigma} i_{o p}-L_{\sigma} \frac{\mathrm{d}}{\mathrm{d} t} i_{o p}-e_{p}+\frac{v_{B}}{2} \\
v_{L} & =R_{\sigma} i_{o n}+L_{\sigma} \frac{\mathrm{d}}{\mathrm{d} t} i_{o n}+e_{n}-\frac{v_{B}}{2} \\
v_{L} & =-R_{\sigma} i_{o p}-L_{\sigma} \frac{\mathrm{d}}{\mathrm{d} t} i_{o p}+L_{m} \frac{\mathrm{d}}{\mathrm{d} t} i_{m p} \\
v_{L} & =-L_{m} \frac{\mathrm{d}}{\mathrm{d} t} i_{m n}+R_{\sigma} i_{o n}+L_{\sigma} \frac{\mathrm{d}}{\mathrm{d} t} i_{o n}
\end{aligned}
$$

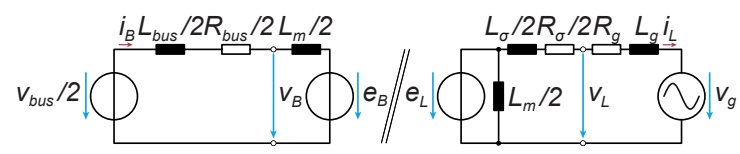

Fig. 11. Equivalent decoupled model for the interleaved stage. 


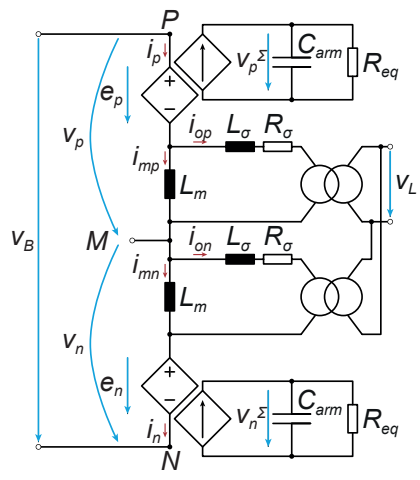

Fig. 12. Stacked MMC stage with equivalent transformer circuit and variables for modeling. $M$ is the transformer's primary side star connection.

It is further simplified to:

$$
\begin{aligned}
v_{L}= & \frac{-e_{p}+e_{n}}{2}+\frac{R_{\sigma}}{2}\left(-i_{o p}+i_{o n}\right) \\
& +\frac{L_{\sigma}}{2}\left(-\frac{\mathrm{d}}{\mathrm{d} t} i_{o p}+\frac{\mathrm{d}}{\mathrm{d} t} i_{o n}\right) \\
v_{L}= & \frac{R_{\sigma}}{2}\left(-i_{o p}+i_{o n}\right)+\frac{L_{\sigma}}{2}\left(-\frac{\mathrm{d}}{\mathrm{d} t} i_{o p}+\frac{\mathrm{d}}{\mathrm{d} t} i_{o n}\right) \\
& +\frac{L_{m}}{2}\left(\frac{\mathrm{d}}{\mathrm{d} t} i_{m p}-\frac{\mathrm{d}}{\mathrm{d} t} i_{m n}\right)
\end{aligned}
$$

As before, several new variables might be advantageously introduced for simplifying the notation:

$$
\begin{array}{ll}
v_{B}=v_{p}+v_{n} & v_{L}=\frac{-v_{p}+v_{n}}{2} \\
e_{B}=e_{p}+e_{n} & e_{L}=\frac{-e_{p}+e_{n}}{2} \\
i_{B}=\frac{i_{p}+i_{n}}{2} & i_{L}=i_{p}-i_{n}-i_{m}=i_{\text {op }}-i_{\text {on }}
\end{array}
$$

This set of equations might be summarized into:

$$
\begin{aligned}
{\left[\begin{array}{c}
v_{B} \\
v_{L}^{\prime} \\
v_{L}^{\prime}
\end{array}\right]=} & {\left[\begin{array}{c}
e_{B} \\
e_{L} \\
0
\end{array}\right]+\left[\begin{array}{ccc}
L_{m} / 2 & 0 & 0 \\
0 & -R_{\sigma} / 2 & 0 \\
0 & -R_{\sigma} / 2 & 0
\end{array}\right]\left[\begin{array}{c}
i_{B} \\
i_{L} \\
i_{m}
\end{array}\right] } \\
& +\left[\begin{array}{ccc}
L_{m} / 2 & 0 & 0 \\
0 & -L_{\sigma} / 2 & 0 \\
0 & -L_{\sigma} / 2 & L_{m} / 2
\end{array}\right] \frac{\mathrm{d}}{\mathrm{d} t}\left[\begin{array}{c}
i_{B} \\
i_{L} \\
i_{m}
\end{array}\right]
\end{aligned}
$$

which is exactly the same set of equations as for the interleaved case, except some change of definition for the bus-side quantities. The similarity between the two cases is highlighted (compare Eq. (19) with Eq. (25), although with different variable definitions). The obtained decoupled model (Fig. 13) is very similar to the one for the interleaved stage, which might be expected, as they are based on the same transformer arrangement. Once again, the same comments regarding the cancellation of DC and AC are valid. Here, the variables defined in Eq. (24) are used.

\section{Control SYSTEM DESIGN}

The control structure is presented in Fig. 14. It is based on the Circulating Current Suppression Controller [19], where the control objectives for the circulating current are to cancel the harmonics and to track the DC current reference $i_{\text {circ }, 0}^{\star}$. Consequently, the arm current is expected to contain only a DC and a fundamental frequency component. As a result, the control system contains two main elements: i) the grid current control that sets $e_{L}$ and ii) the balancing control through imposition of a DC circulating current via the summed capacitor voltage control (total energy controller). The second harmonic in the summed capacitor voltages are notch filtered, as the total energy controller sets a DC circulating current reference.

Please note that while it is tempting to set the 6 arm currents individually for the interleaved case, so that the stored energy would be perfectly controlled, the DC cancellation in the transformer cannot be anymore guaranteed in this scenario.

\section{COMPARATIVE PERFORMANCE ASSESSMENT}

\section{A. System parameters}

The converter parameters are indicated in Table I. The value of the arm inductance is not optimized at this stage, as it is beyond the scope of the paper.

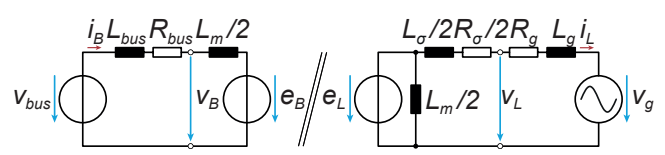

Fig. 13. Equivalent decoupled model for the stacked stage

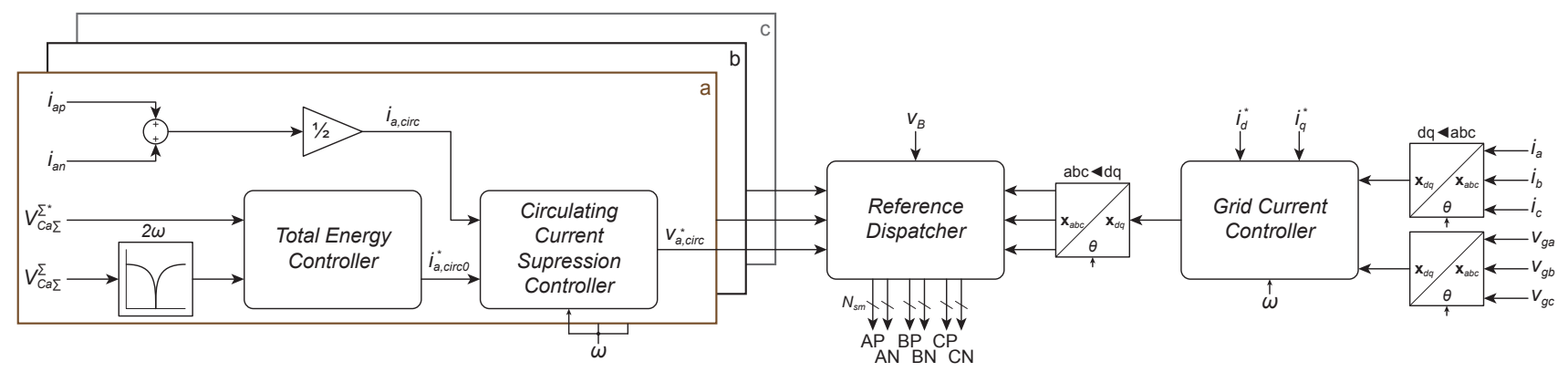

Fig. 14. Control structure: the circulating and grid currents are controlled independently. 
The capacitor sizing relies on the expression of [20], as the whole AC power oscillation has to be buffered at the phase leg level, as no power is exchanged for this purpose between the legs. Considering the parameters from Table I and assuming a voltage ripple of $2 \%, C_{s m}=1.6 \mathrm{mF}$ is calculated.

The transformer magnetizing inductance has been chosen for a $5 \%$ magnetizing current compared to the nominal output current.

\section{B. Simulation results}

The simulation results are presented in Fig. 15. The selected modulation is PS-PWM with $2 N+1$ levels. As in the interleaved case the DC link voltage is half compared to the one for classical and interleaved MMCs, the bus current doubles to maintain the same power transfer.

The plots (a) - (c) show the arm, AC output and DC source currents for the phase $a$. As the arm currents contain only a DC and fundamental (with opposite phase) components,

TABLE I

SYSTEM PARAMETERS

\begin{tabular}{llllll}
\hline$V_{D C}$ & $10 \mathrm{kV}$ & $L_{\text {bus }}$ & $100 \mu \mathrm{H}$ & $R_{\text {bus }}$ & $100 \mu \Omega$ \\
$N_{s m}$ & 6 & $\varepsilon_{\Delta V_{s m}}$ & $2 \%$ & $C_{\text {sm }}$ & $1.6 \mathrm{mF}$ \\
$L_{\text {arm }}$ & $1.5 \mathrm{mH}$ & $R_{\text {arm }}$ & $10 \mathrm{~m} \Omega$ & $L_{m}$ & $1.5 \mathrm{H}$ \\
$L_{g}$ & $5 \mathrm{mH}$ & $R_{g}$ & $100 \mathrm{~m} \Omega$ & $m$ & 0.95 \\
$P_{\text {nom }}$ & $1 \mathrm{MW}$ & $f_{\text {sw }}$ & $1 \mathrm{kHz}$ & $n$ & 1 \\
\hline
\end{tabular}

the circulating current will be AC free. The CCSC works as expected.

The plots (d) - (f) show that the resulting current in the magnetic material (i.e. simply the magnetizing current for the classical MMC, or the difference between the two magnetizing currents for the interleaved and stacked MMCs) does not contain any DC component. In other words, no DC flux is present in the magnetic material. This also means that the DC cancellation is effective, as a result of the proposed winding configuration.

In the plots $(\mathrm{g})$ - (i), the summed capacitor voltages remain stable with an average value of $12 \mathrm{kV}$.

\section{CONCLUSION}

Generalized topologies of galvanically isolated MMC, using single-stage DC/AC conversion as an example, can be realized using either interleaved or stacked structures, and have shown close similarities with the classical MMC case. From a control point of view, there is no fundamental difference due to the integration of the transformer at the arm level. The simulation results have highlighted the effectiveness of the DC bias cancellation in the transformer.

Future work will assess the impact of the LFT integration on the overall system design, with a focus on weight, volume and efficiency.

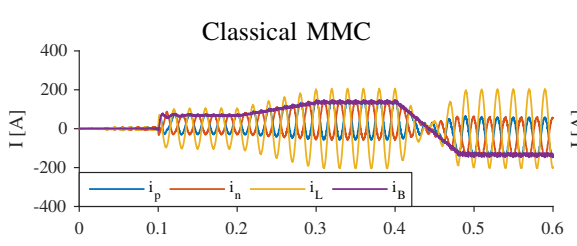

(a)

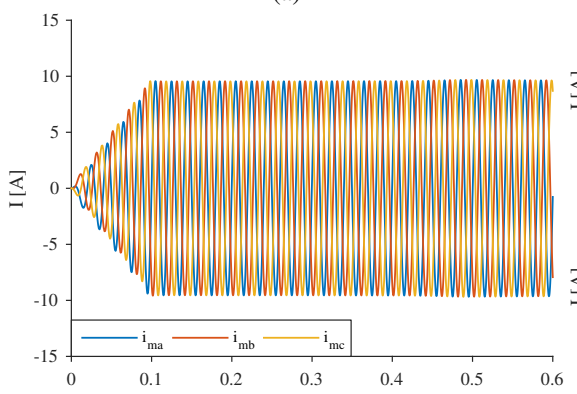

(d)

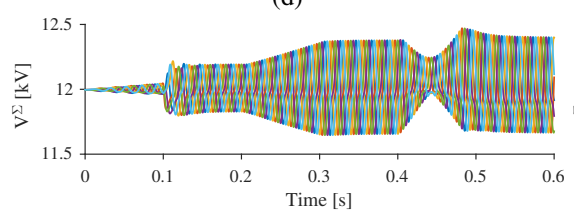

(g)

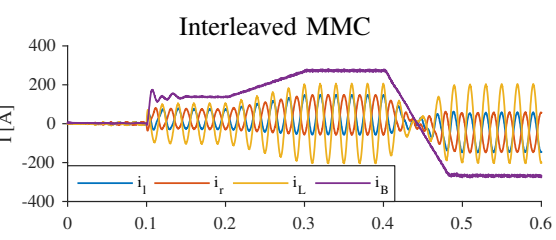

(b)
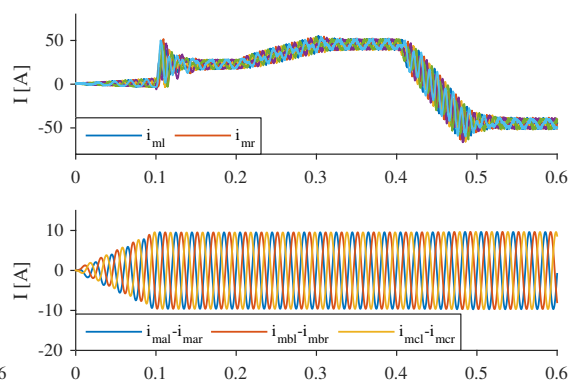

(e)

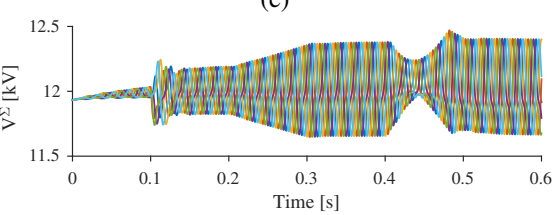

(h)

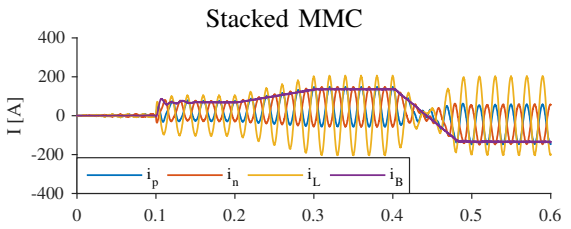

(c)
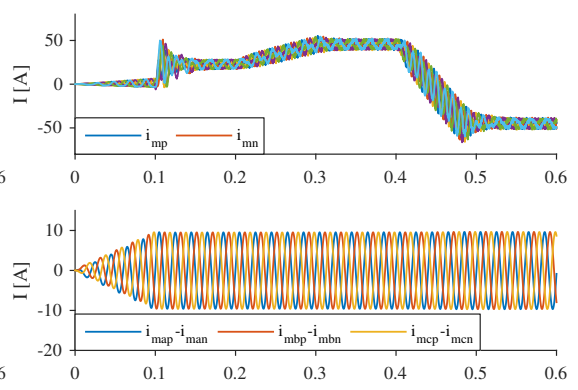

(f)

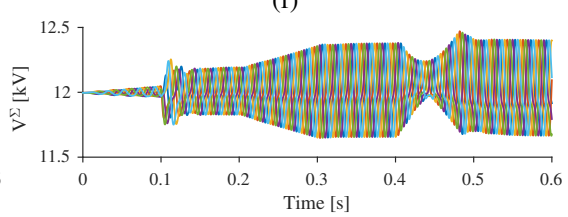

(i)

Fig. 15. Simulation results: (a)-(c) arm currents, output current and bus current, (d)-(f) magnetizing currents and the image of the magnetizing flux through the transformer without DC, (g)-(i) summed capacitor voltages. For $t \in\{0 ; 0.1\}[\mathrm{s}]$ the transformer is magnetized. At $t=0.1 \mathrm{~s}$ the $i_{d, \text { ref }}$ is changed from 0 to $100 \mathrm{~A}$. For $t \in\{0.2 ; 0.3\}[\mathrm{s}] i_{d, r e f}$ is ramped up to $200 \mathrm{~A}$. For $t \in\{0.4 ; 0.48\}[\mathrm{s}]$ there is a full power reversal. Please note that $v_{B}$ is identical for both classical and stacked MMC, while only half for interleaved MMC, explaining the double bus current value for the latter case. 


\section{ACKNOWLEDGEMENT}

This work is part of the Swiss Competence Centers for Energy Research (SCCER) initiative which is supported by the Swiss Commission for Technology and Innovation (CTI).

\section{REFERENCES}

[1] A. Lesnicar and R. Marquardt, "An innovative modular multilevel converter topology suitable for a wide power range," in Power Tech Conference Proceedings, 2003 IEEE Bologna, vol. 3, Jun. 2003, 6 pp. Vol.3.

[2] J. Kolb, F. Kammerer, and M. Braun, "Straight forward vector control of the modular multilevel converter for feeding three-phase machines over their complete frequency range," in IECON 2011 - 37th Annual Conference on IEEE Industrial Electronics Society, Nov. 2011, pp. 1596-1601.

[3] K. Ilves, L. Bessegato, and S. Norrga, "Comparison of cascaded multilevel converter topologies for ac/ac conversion," in Power Electronics Conference (IPECHiroshima 2014 - ECCE-ASIA), 2014 International, May 2014, pp. 1087-1094.

[4] M. Hiller, D. Krug, R. Sommer, and S. Rohner, "A new highly modular medium voltage converter topology for industrial drive applications," in Power Electronics and Applications, 2009. EPE '09. 13th European Conference on, Sep. 2009, pp. 1-10.

[5] G. Karady, A. Huang, and M. Baran, "Freedm system: An electronic smart distribution grid for the future," in Transmission and Distribution Conference and Exposition (T D), 2012 IEEE PES, May 2012, pp. 1-6.

[6] M. Hagiwara and H. Akagi, "Experiment and simulation of a modular push-pull pwm converter for a battery energy storage system," Industry Applications, IEEE Transactions on, vol. 50, no. 2, pp. 1131-1140, Mar. 2014.

[7] High voltage dc/dc converter with transformer driven by modular multilevel converters ( $\mathrm{mmc}$ ), WO Patent App. PCT/ EP2011/ 070, 629, May 2013.

[8] A. Das, H. Nademi, and L. Norum, "A new circuit topology of modular multilevel converter $(\mathrm{mmc})$ with an open end transformer," in PCIM Europe 2012; International Exhibition and Conference for Power Electronics, Intelligent Motion, Renewable Energy and Energy Management; Proceedings of, May 2012.

[9] Multilevel converter, WO Patent App. PCT/EP2012/072,757, Jan. 2014.

[10] S. Tamada, Y. Nakazawa, and S. Irokawa, "A proposal of modular multilevel converter applying three winding transformer," in Power Electronics Conference (IPECHiroshima 2014 - ECCE-ASIA), 2014 International, May 2014, pp. 1357-1364.
[11] L. Baruschka, D. Karwatzki, M. von Hofen, and A. Mertens, "A new modular multilevel ac/dc converter topology applied to a modular multilevel $\mathrm{dc} / \mathrm{dc}$ converter," in Power Electronics and Applications (EPE'14ECCE Europe), 2014 16th European Conference on, Aug. 2014, pp. 1-10.

[12] N. Cherix, M. Vasiladiotis, and A. Rufer, "Functional modeling and energetic macroscopic representation of modular multilevel converters," in Power Electronics and Motion Control Conference (EPE/PEMC), 2012 15th International, Sep. 2012.

[13] A. Rasic, U. Krebs, H. Leu, and G. Herold, "Optimization of the modular multilevel converters performance using the second harmonic of the module current," in Power Electronics and Applications, 2009. EPE '09. 13th European Conference on, Sep. 2009, pp. 1-10.

[14] S. Engel and R. De Doncker, "Control of the modular multi-level converter for minimized cell capacitance," in Power Electronics and Applications (EPE 2011), Proceedings of the 2011-14th European Conference on, Aug. 2011, pp. 1-10.

[15] M. Winkelnkemper, A. Korn, and P. Steimer, "A modular direct converter for transformerless rail interties," in Industrial Electronics (ISIE), 2010 IEEE International Symposium on, Jul. 2010, pp. 562-567.

[16] A. Christe and D. Dujić, "State-space modeling of modular multilevel converters including line frequency transformer," in Power Electronics and Applications (EPE), 2015 17th European Conference on, 2015.

[17] A. Antonopoulos, L. Angquist, and H.-P. Nee, "On dynamics and voltage control of the modular multilevel converter," in Power Electronics and Applications, 2009. EPE '09. 13th European Conference on, Sep. 2009, pp. 1-10.

[18] S. Rohner, J. Weber, and S. Bernet, "Continuous model of modular multilevel converter with experimental verification," in Energy Conversion Congress and Exposition (ECCE), 2011 IEEE, Sep. 2011, pp. 4021-4028.

[19] Q. Tu, Z. Xu, and L. Xu, "Reduced switching-frequency modulation and circulating current suppression for modular multilevel converters," Power Delivery, IEEE Transactions on, vol. 26, no. 3, pp. 2009-2017, Jul. 2011.

[20] A. Lesnicar and R. Marquardt, "A new modular voltage source inverter topology," in Proc. of the 10th European Conference on Power Electronics and Applications (EPE), 2003. 